\title{
VIRIDANTHA, UN GÉNERO NUEVO DE BROMELIACEAE (TILLANDSIOIDEAE) ENDÉMICO DE MÉXICO
}

\author{
Adolfo Espejo-Serna \\ Herbario Metropolitano \\ Departamento de Biología, C.B.S. \\ Universidad Autónoma Metropolitana \\ Unidad Iztapalapa \\ Apartado postal $55-535$ \\ 09340 México, D.F. \\ email: aes@xanum.uam.mx
}

\begin{abstract}
RESUMEN
Se propone como nuevo para la ciencia el género Viridantha Espejo (Bromeliaceae), constituido por dos secciones y seis especies anteriormente adscritas a Tillandsia, endémicas de México; el género se caracteriza por presentar estambres todos iguales en longitud, filamentos aplanados, anteras de 2 a $4 \mathrm{~mm}$ de largo, subbasifijas, pétalos ligulados, libres, de color verde oscuro, flores proterandras, dísticas, escamas largamente asimétricas, densa a muy densamente dispuestas y estigmas del tipo simple-erecto (tipo I, sensu Brown \& Gilmartin).
\end{abstract}

Palabras clave: Bromeliaceae, México, Tillandsia plumosa, Viridantha.

\begin{abstract}
The genus Viridantha Espejo (Bromeliaceae) with two sections and six species, all endemic to Mexico and previously treated as Tillandsia, is proposed as new. Viridantha is characterized by the stamens equal in length, flat filaments and subbasifixed anthers, 2 - $4 \mathrm{~mm}$ long; ligulate, free, dark green petals; distichous, protandrous flowers; long asymmetric, densely to very densely disposed scales, and simple-erect stigmas (type I, sensu Brown \& Gilmartin).
\end{abstract}

Key words: Bromeliaceae, Mexico, Tillandsia plumosa, Viridantha.

\section{INTRODUCCIÓN}

Las Tillandsioideae (Bromeliaceae) presentan como únicos caracteres en común el ovario súpero, o casi así y sus frutos capsulares, dehiscentes, conteniendo semillas plumoso-apendiculadas (Baker, 1889; Mez, 1896; Smith \& Downs, 1977; Till, 2000a, b). Por lo demás, constituyen un grupo heterogéneo que incluye especies muy diferentes entre sí, tanto en los caracteres vegetativos como en los florales. Lo anterior es especialmente evidente en el complejo Tillandsia-Vriesea, en donde encontramos plantas que miden desde unos cuantos centímetros de alto, como $T$. ionantha Planch. hasta 
verdaderos gigantes como $T$. grandis Schltdl., o plantas densamente escamosas y cinéreas como $T$. plumosa Baker hasta especies prácticamente glabras como $T$. leiboldiana Schltdl. o V. heliconioides (Kunth) Hook. ex Walp. En cuanto a la morfología floral, podemos encontrar diferencias en el tamaño y el color de las flores, el tamaño y la posición de los filamentos y las anteras, la disposición de los sépalos y los pétalos, la orientación de las flores en la antesis, etc., caracteres que indudablemente están relacionados con el proceso de polinización y la biología reproductiva de las especies y que por lo tanto influyen en su evolución y en sus relaciones filogenéticas.

La clasificación infragenérica en Tillandsia ha sido objeto de estudio por diversos especialistas en la familia, sin embargo no se ha logrado todavía un arreglo satisfactorio. Los subgéneros propuestos por autores como Baker (1889) y Mez (1896) (Allardtia, Tillandsia, Strepsia, Diaphoranthema, Platystachys, Anoplophytum, Pseudocatopsis, etc.) se basan en caracteres únicos como son la presencia o ausencia de escamas en los pétalos, las anteras erectas o versátiles o la condición exserto-inclusa de los estambres y/o estilo, o bien en caracteres tan poco claros como la variación foliar expresada por Baker (1889) como "differs ... only in leaf". Smith y Downs (1977) retomaron en su trabajo las propuestas de Baker y Mez y en algunos casos situaron especies fuera de lugar debido a que nunca vieron plantas vivas y por lo tanto no pudieron examinar las flores, lo cual aumentó el caos en las delimitaciones genéricas y/o subgenéricas.

La necesidad de revisar la circunscripción de los géneros y subgéneros dentro de las Tillandsioideae, utilizando para ello tanto caracteres florales como vegetativos, ha sido expresada por algunos autores (Gardner, 1982, 1986; Smith \& Pittendrigh, 1953) y otros más, haciendo uso de los mismos, han llegado a segregar grupos definiendo géneros nuevos o restableciendo nombres genéricos anteriormente propuestos, como Alcantarea, Werahuia (Grant, 1995) y Racinaea (Spencer \& Smith,1993).

Gardner (1982, 1986) revisó las especies mexicanas de Tillandsia subgénero Tillandsia (sensu Smith \& Downs, 1977, pero incluyendo algunas del subgénero Allardtia), utilizando caracteres florales tomados de plantas vivas (79 especies de las 123 consideradas) y caracteres de los especímenes herborizados. Como resultado de su trabajo, propuso una división preliminar del subgénero en cinco grupos, sin dar nombre formal a ninguno de ellos.

Es claro que la clasificación genérica hasta ahora usada para el complejo Tillandsia-Vriesea deja mucho que desear y que, por otra parte, existen grupos que pueden ser delimitados por un conjunto de caracteres coherentes y consistentes, tanto florales como vegetativos, que reflejen mejor las relaciones filogenéticas y permitan definir un arreglo taxonómico más natural.

Uno de los grupos que Gardner $(1982,1986)$ reconoció en su trabajo es el de Tillandsia plumosa Baker y, aunque ella misma señaló que no corresponde estrictamente a Tillandsia subgénero Tillandsia, lo incluyó en su estudio, debido a que Smith y Downs (1977) habían considerado a dos de las seis especies del grupo: T. ehrenbergii Klotzsch ex Beer (= T. tortilis Klotzsch ex Baker) y T. lepidosepala L. B. Sm. dentro de dicho subgénero.

Los otros cuatro taxa del complejo fueron ubicados por los autores ya mencionados (Smith \& Downs, 1977) en Tillandsia subgénero Allardtia y constituyen un ejemplo más de lo inadecuado de la clasificación subgenérica en Tillandsia porque, aunque todas ellas presentan estambres inclusos, en el resto de sus características 
difieren notablemente de las propuestas como diagnósticas para el subgénero (Baker, 1889; Mez, 1896).

Tillandsia subgénero Tillandsia, tipificado por Tillandsia polystachya (L.) L. (Renealmia polystachya L.), se caracteriza por sus flores generalmente erectas, protóginas, con los pétalos espatulados, violáceos o verde-amarillentos, muy rara vez amarillos, rojos o blancos, de 4 a $6.8 \mathrm{~cm}$ de largo, constreñidos hacia el ápice alrededor de los estambres formando una garganta, por sus estambres exsertos, dispuestos en dos series de distinto tamaño, con los filamentos aplanados y ensanchados en el ápice y sus anteras versátiles. Además, las plantas del grupo presentan generalmente rosetas infundifbuliformes de tipo tanque y son por lo común glabras a glabrescentes (cf. Gardner, 1982, 1986).

En tanto que los caracteres que delimitan al grupo de $T$. plumosa son los siguientes: flores proterandras, dísticas, descendentes, con los pétalos ligulados, de color verde oscuro, de 2 a $3 \mathrm{~cm}$ de largo, no constreñidos para formar una garganta, estambres inclusos, todos iguales en longitud, con los filamentos aplanados, las anteras subbasifijas y el estigma del tipo simple-erecto (tipo I, sensu Brown \& Gilmartin, 1984). Las rosetas en este conjunto son de tipo compacto, no forman tanque y tienden a la caulescencia, además de presentar escamas largamente asimétricas, densa a muy densamente dispuestas.

El grupo de $T$. plumosa se distingue así muy claramente de las demás especies de Tillandsia y presenta con el resto del complejo Tillandsia-Vriesea mayores diferencias que las existentes entre géneros como Vriesea y Tillandsia, Racinaea y Tillandsia o aun entre subfamilias como Tillandsioideae y Pitcairnioideae.

Cabe indicar aquí que algunas especies sudamericanas entre las que pueden citarse T. tectorum E. Morren, T. heteromorpha Mez, T. vernicosa Baker, T. didisticha (E. Morren) Baker y $T$. comarapensis $\mathrm{H}$. Luther, comparten con el grupo de $T$. plumosa algunas características, como los estambres inclusos y del mismo largo, la forma y el tamaño de los pétalos, el denso indumento y la tendencia a la caulescencia, aunque difieren del mismo por presentar flores erectas, pétalos blancos o violáceos con el ápice blanco y estambres plicados.

Con base en lo anteriormente expuesto, se propone como nuevo el siguiente género:

Viridantha Espejo, genus novum

Herbae acaules vel caulescentes, epiphyticae vel rupicolae. Folia linearia. Squamae longialatae, asymmetricae. Inflorescentia pedunculata vel nidularis, simplex vel composita. Spicae applanatae. Flores distichi, proterandri, actinomorphi. Petala ligulata, libera, nuda, atroviridia. Stamina aequilonga, filamentis applanatis, antherae $2-4 \mathrm{~mm}$ longae, subbasifixae vel erectae. Stamina et pistillum in corolla inclusa. Stigma classi I teste Brown et Gilmartin. Ovarium superum. Capsula oblonga, acuminata, septicida. Semina basi appendiculata.

Hierbas acaules o caulescentes, epífitas o rupícolas, solitarias a rara vez cespitosas, xerófilas, ageotrópicas, de menos de $35 \mathrm{~cm}$ de alto. Hojas lineares. Escamas largamente aladas, asimétricas. Inflorescencia pedunculada a nidular, simple o compues- 
ta. Espigas aplanadas. Flores dísticas, proterandras, horizontales a péndulas, actinomorfas, tubulares. Pétalos ligulados, libres, sin escamas, de color verde oscuro. Estambres todos del mismo largo, con los filamentos aplanados, anteras de 2 a $4 \mathrm{~mm}$ de largo, subbasifijas a erectas, amarillas o raramente negras. Estambres y pistilo más cortos que los pétalos, incluidos en la corola. Estigma del tipo simple-erecto (Tipo I, Brown \& Gilmartin, 1984). Ovario súpero. Cápsula oblonga, acuminada, septicida, de color verde oscuro. Semillas con un apéndice plumoso en la base.

TYPUS: Viridantha plumosa (Baker) Espejo

El género es endémico del centro de México, donde se distribuye en los estados de Aguascalientes, Durango, Guanajuato, Guerrero, Hidalgo, Jalisco, México, Michoacán, Morelos, Oaxaca, Puebla, Querétaro, San Luis Potosí y Zacatecas (Figs. 2, 3 y 5).

El nombre genérico hace alusión al característico color verde oscuro que presentan las flores de las especies del grupo.

Clave para la identificación de las secciones y de las especies del género Viridantha

1 Rosetas regulares, más o menos esféricas en contorno, acaules o, si son caulescentes, el tallo de más de $1 \mathrm{~cm}$ de diámetro; hojas numerosas

Viridantha sección Viridantha

2 Inflorescencia escaposa, generalmente más larga que las hojas.

3 Inflorescencia compuesta, globosa V. plumosa

3 Inflorescencia simple, raramente biespigada, aplanada V. ignesiae

2 Inflorescencia nidular, siempre más corta que las hojas.

4 Hojas de menos de $2 \mathrm{~mm}$ de ancho; anteras amarillas; plantas generalmente epifíticas V. atroviridipetala

4 Hojas de más de 3.5 mm de ancho; anteras negras; plantas rupícolas

V. mauryana

1 Rosetas irregulares, amorfas en contorno, caulescentes, el tallo de menos de $0.5 \mathrm{~cm}$ de diámetro; hojas pocas Viridantha sección Caulescens

5 Inflorescencia escaposa, generalmente más larga que las hojas, el pedúnculo de menos de $2 \mathrm{~mm}$ de diámetro V. tortilis

5 Inflorescencia nidular 0 , si es escaposa, con el pedúnculo de más de $2.5 \mathrm{~mm}$ de diámetro y siempre más corta que las hojas V. lepidosepala

\section{Viridantha sectio Viridantha} simplici.

Herbae acaules, foliis numerosis, rosulatis, inflorescentia composita vel rarius

Viridantha atroviridipetala (Matuda) Espejo, comb. nov. Basiónimo: Tillandsia atroviridipetala Matuda, Cact. Suc. Mex. 2: 53-54, f. 40. 1957. TIPO: Estado de México, 
Puente de Calderón, en orilla de arroyo, epífita en Taxodium mucronatum, 17.X.1955, E. Matuda 32632 (Holotipo: MEXU (probablemente no existe ejemplar)). Fig. 1.

De acuerdo con el protólogo, el holotipo debería estar depositado en el Herbario Nacional (MEXU); sin embargo, no fue encontrado aun después de una revisión exhaustiva de la colección de bromeliáceas, por lo que es muy probable que no exista ejemplar alguno. Tampoco se sabe de la existencia de isotipos, al menos en los principales herbarios mexicanos (CHAPA, ENCB, IBUG, IEB, UAMIZ, XAL) y norteamericanos (F, HUH, NY, US). De igual modo, ha sido imposible localizar el paratipo (Matuda 32633) citado en la publicación original.

Por otra parte, existen dos duplicados del ejemplar E. Matuda 30414, recolectado en la localidad tipo, depositados en el Herbario Nacional (MEXU 128965!, MEXU 141734!). Cabe señalar que el Dr. Matuda tenía una manera muy peculiar de trabajar su material y como es sabido, existen innumerables inconsistencias en los datos, la numeración y la ubicación física de sus especímenes designados como tipos.

Debido a lo anterior y de acuerdo con el artículo 9, incisos 9.1 y 9.9 del Código Internacional de Nomenclatura Botánica, se propone aquí el siguiente neotipo: Hidalgo, 5 km al E de Metzquititlán, J. Rzedowski 19504 (Neotipo (aquí designado): ENCB!; Isoneotipos: MEXU!, MICH!)

V. atroviridipetala se conoce de los estados de Guanajuato, Guerrero, Hidalgo, Jalisco, México, Michoacán, Morelos, Oaxaca, Puebla y Zacatecas. Fig. 2.

Viridantha ignesiae (Mez) Espejo, comb. nov. Basiónimo: Tillandsia ignesiae Mez, Bull. Herb. Boissier sér. 2. 3: 143. 1903. TIPO: Michoacán, municipio La Huacana, Monte de Santa Inés "Ignes" [ca. 6-7 km NE de Inguarán] (fleurs) et de Las Cieneguillas "Seneguías" (fruits), 1500 m, 4.IV.1898, E. Langlassé 93 (fleurs) (Holotipo: B ?; Isotipos: G!, GH!, K, P!, P, US 385728!).

Tillandsia lecomtei Poiss. \& P. Menet, Bull. Mus. Hist. Nat. (Paris) 14: 236-237. 1908, nomen superfluum. TIPO: Michoacán, municipio La Huacana, Monte de Santa Inés "Ignes" [ca. 6-7 km NE de Inguarán] (fleurs) et de Las Cieneguillas "Seneguías" (fruits), 1500 m, 4.IV.1898, E. Langlassé 93 (Holotipo: P!; Isotipos: B?, G!, GH!, K, P, US 385728!). Fig. 2.

V. ignesiae se conoce de los estados de Guerrero, Jalisco, México y Michoacán.

Viridantha mauryana (L. B. Sm.) Espejo, comb. nov. Basiónimo: Tillandsia mauryana L. B. Sm., Contr. Gray Herb. 117: 31, t. 2, f. 32, 33. 1937. TIPO: Hidalgo, cañada de Metztitlán, 1300-1500 m, 17.IV.1891, P. Maury 5747 (Holotipo: GH!; Isotipos: F, GH!).

Existen en el Gray Herbarium (GH) dos duplicados de la colecta de Maury, ambos anotados por Smith. El holotipo, sin embargo, tiene escrita la palabra "TYPE", por lo que no hay lugar a dudas sobre cuál espécimen es el holotipo y cuál es el duplicado.

V. mauryana se conoce de los estados de Hidalgo, Jalisco, Oaxaca y Zacatecas. Fig. 3. 


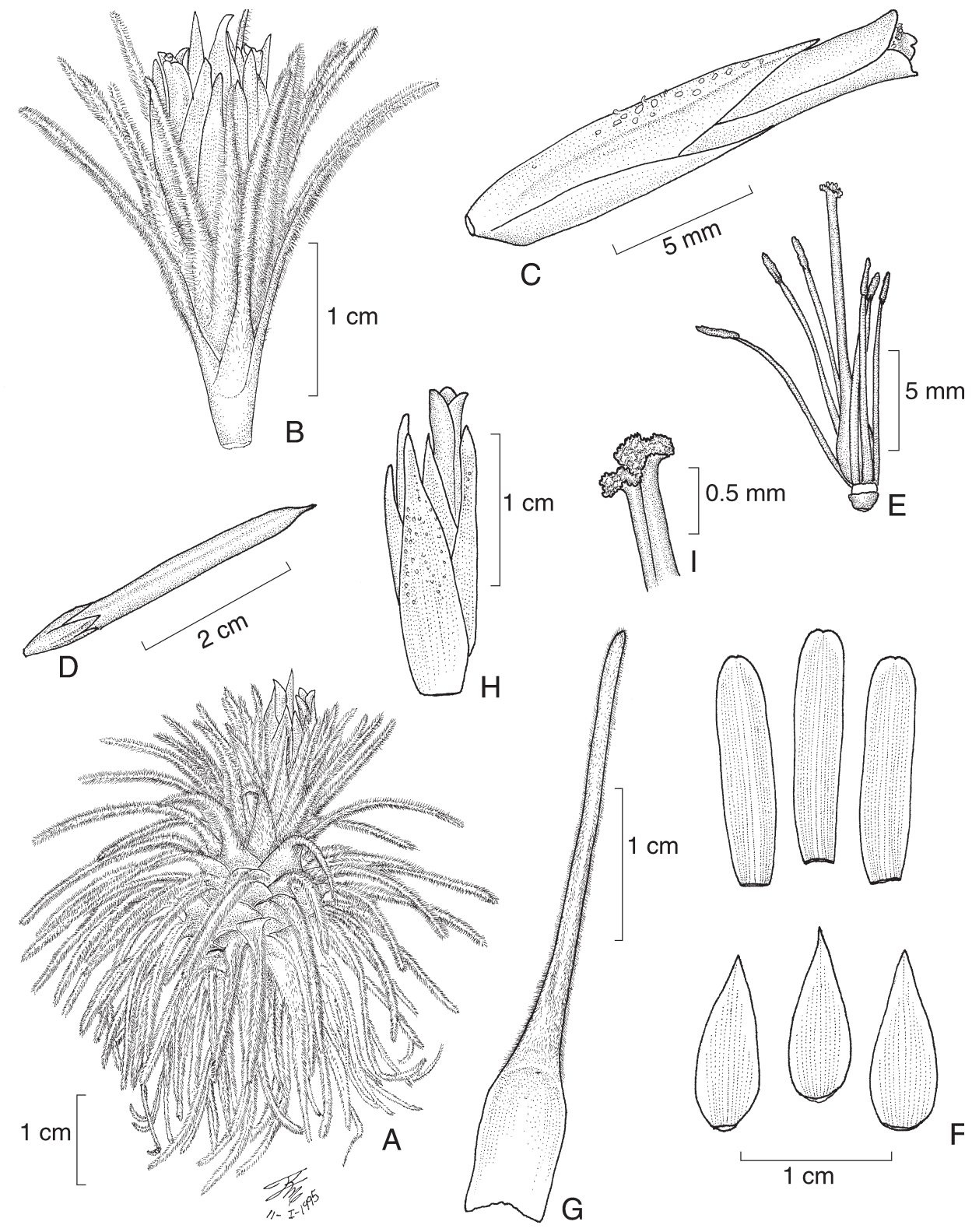

Fig. 1. Viridantha atroviridipetala (Matuda) Espejo. A. Hábito; B. Inflorescencia; C. Flor; D. Fruto; E. Detalle del androceo y el gineceo; F. Pétalos y sépalos; G. Hoja; H. Detalle de una espiga; I. Estigma. 


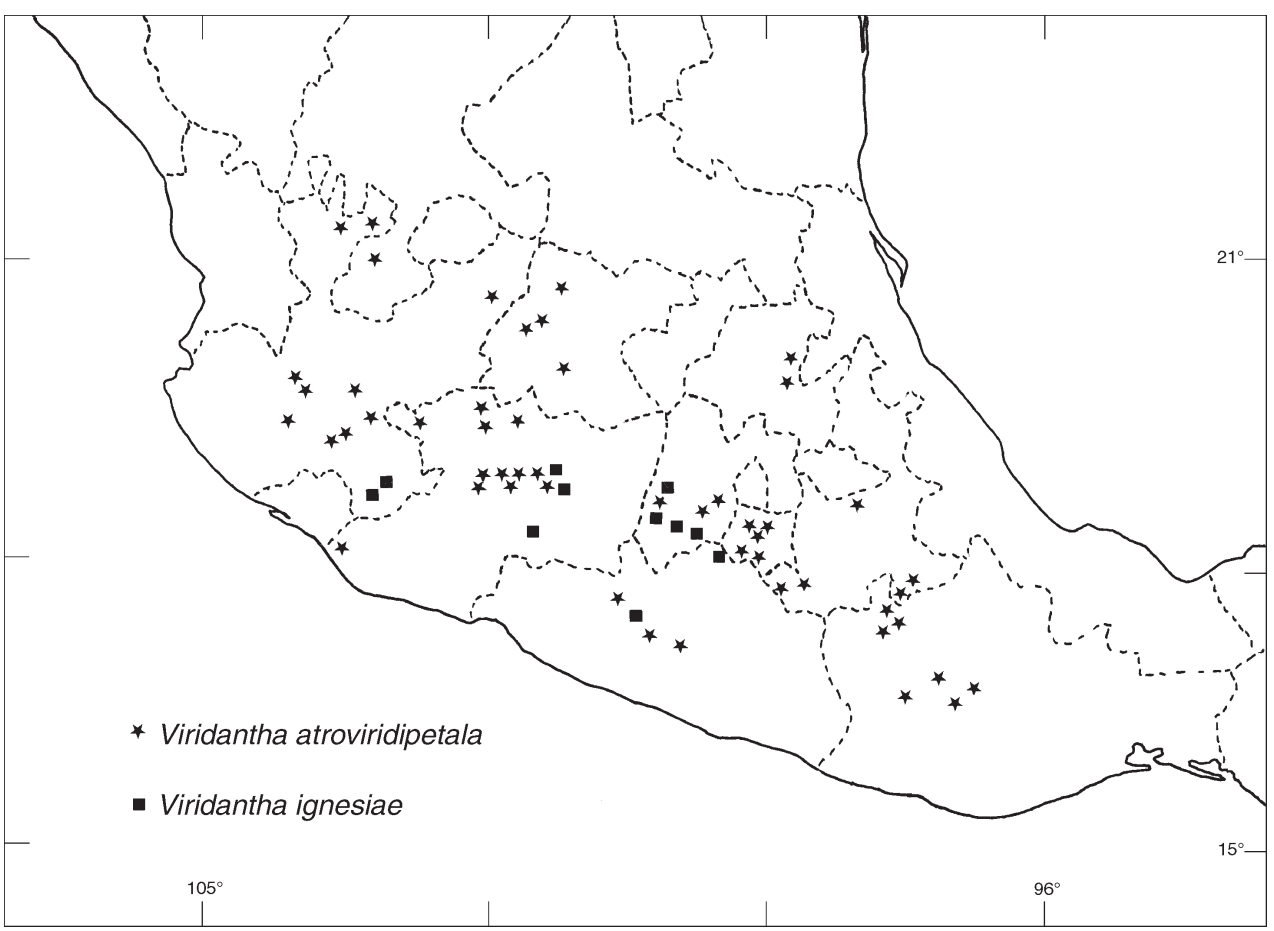

Fig. 2. Distribución conocida de Viridantha atroviridipetala (Matuda) Espejo y V. ignesiae (Mez) Espejo.

Viridantha plumosa (Baker) Espejo, comb. nov. Basiónimo: Tillandsia plumosa Baker, J. Bot. 26: 13. 1888. TIPO: Puebla, G. Andrieux 57 (Holotipo: K foto!; Isotipo: M). Fig. 5.

V. plumosa se conoce de los estados de Guerrero, México, Oaxaca y Puebla.

Viridantha sectio Caulescens Espejo, sectio nova

Differt a Viridantha sectione Viridantha caule elongato, foliis paucioribus et inflorescentia simplici.

TYPUS: Viridantha tortilis (Klotzsch ex Baker) Espejo

Viridantha lepidosepala (L. B. Sm.) Espejo, comb. nov. Basiónimo: Tillandsia lepidosepala L. B. Sm., Proc. Amer. Acad. Arts 70 (Contr. Gray Herb. 106): 155, t. 2, f. 2, 


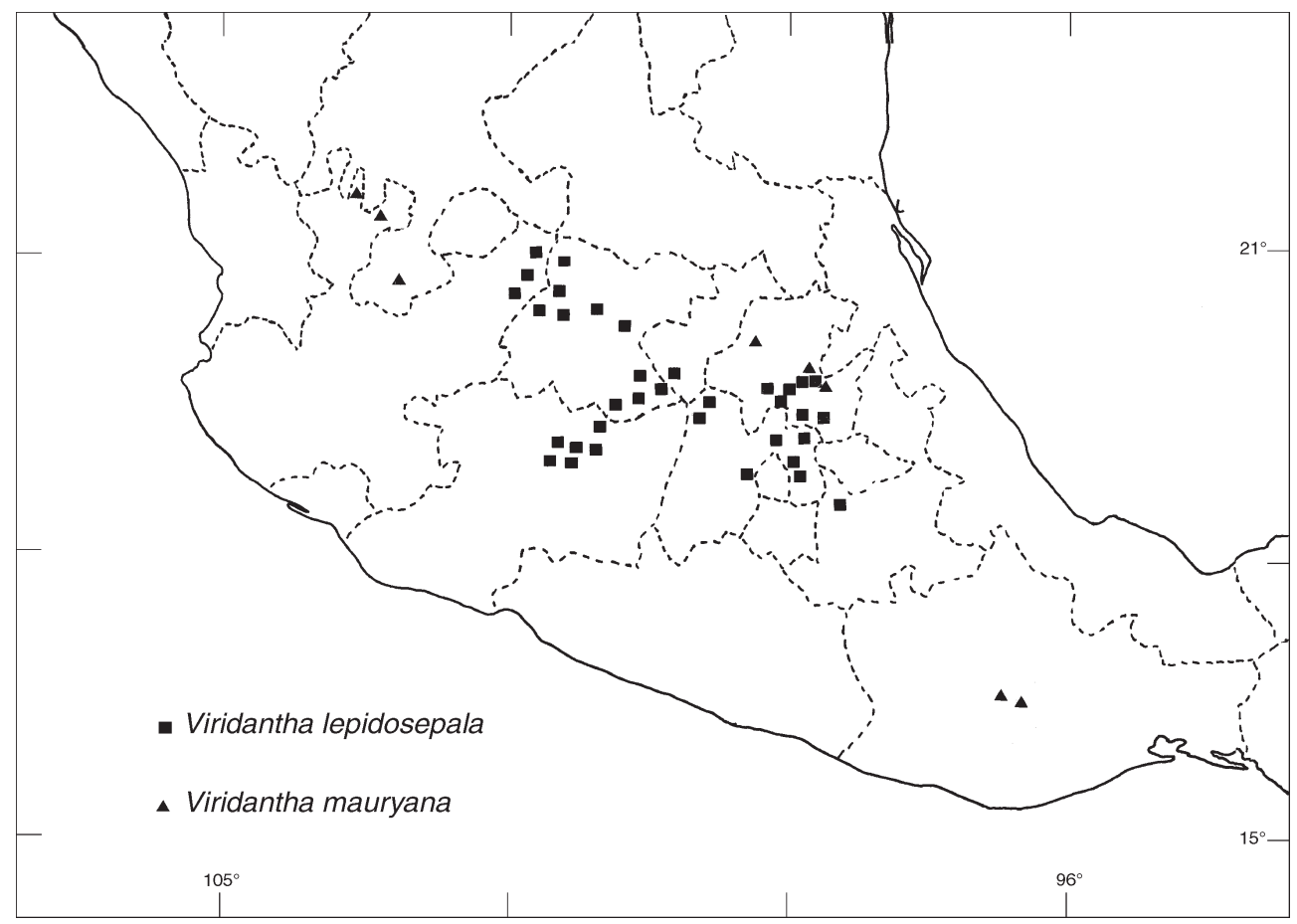

Fig. 3. Distribución conocida de Viridantha lepidosepala (L. B. Sm.) Espejo y V. mauryana (L. B. Sm.) Espejo.

3. 1935. TIPO: Michoacán, on trees near Lake Cuitzeo, 9.VIII.1892, C. G. Pringle 5323 (Holotipo: GH!; Isotipo: VT!).

V. lepidosepala se conoce de los estados de Guanajuato, Hidalgo, Jalisco, México, Michoacán, Puebla y Querétaro. Fig. 3.

Viridantha tortilis (Klotzsch ex Baker) Espejo, comb. nov. Basiónimo: Tillandsia tortilis Klotzsch ex Baker, J. Bot. (London) 25: 237. 1887, non Brongn. ex E. Morren, 1878, nomen. TIPO: San Luis Potosí, Central Mexico, chiefly in the region of San Luis Potosí, $22^{\circ} \mathrm{N}, 6000-8000 \mathrm{ft}[1800-2400 \mathrm{~m}], 1878$, C. C. Parry \& E. Palmer 872 (Lectotipo (designado por Till, 1986): BM; Isolectotipos: E, GH!, K, P!, US). Fig. 4.

Tillandsia ehrenbergii Klotzsch ex Beer, Fam. Bromel. 264. 1857, nomen nudum. Platystachys ehrenbergii Beer, Fam. Bromel. 264. 1857, pro syn. TIPO: Hidalgo, prope 
Espejo-Serna: Viridantha, Un Género Nuevo de Bromeliaceae Endémico de México

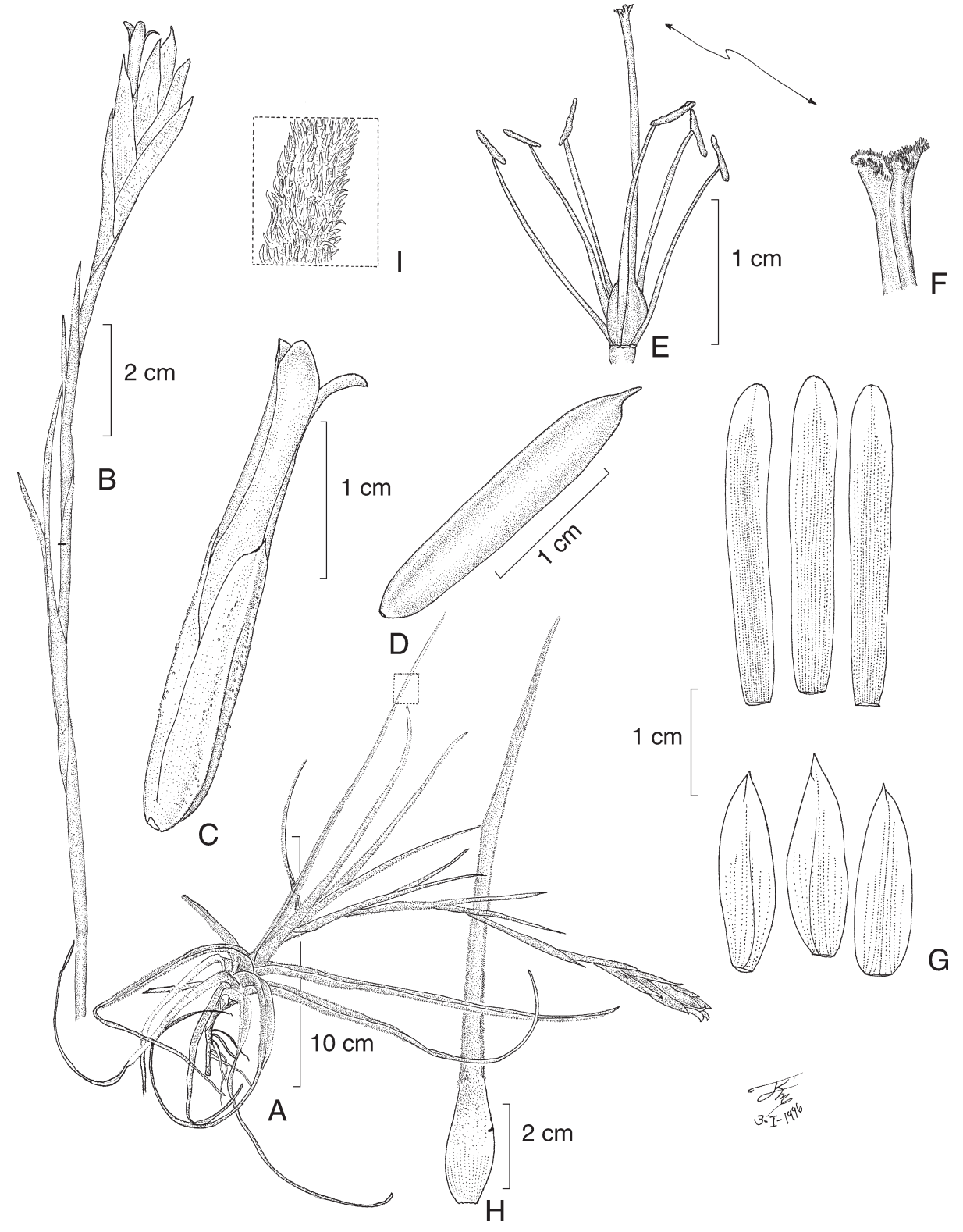

Fig. 4. Viridantha tortilis (Klotzsch ex Baker) Espejo. A. Hábito; B. Inflorescencia; C. Flor; D. Fruto; E. Detalle del androceo y el gineceo; F. Estigma; G. Pétalos y sépalos; H. Hoja; I. Detalle de escamas en la hoja. 


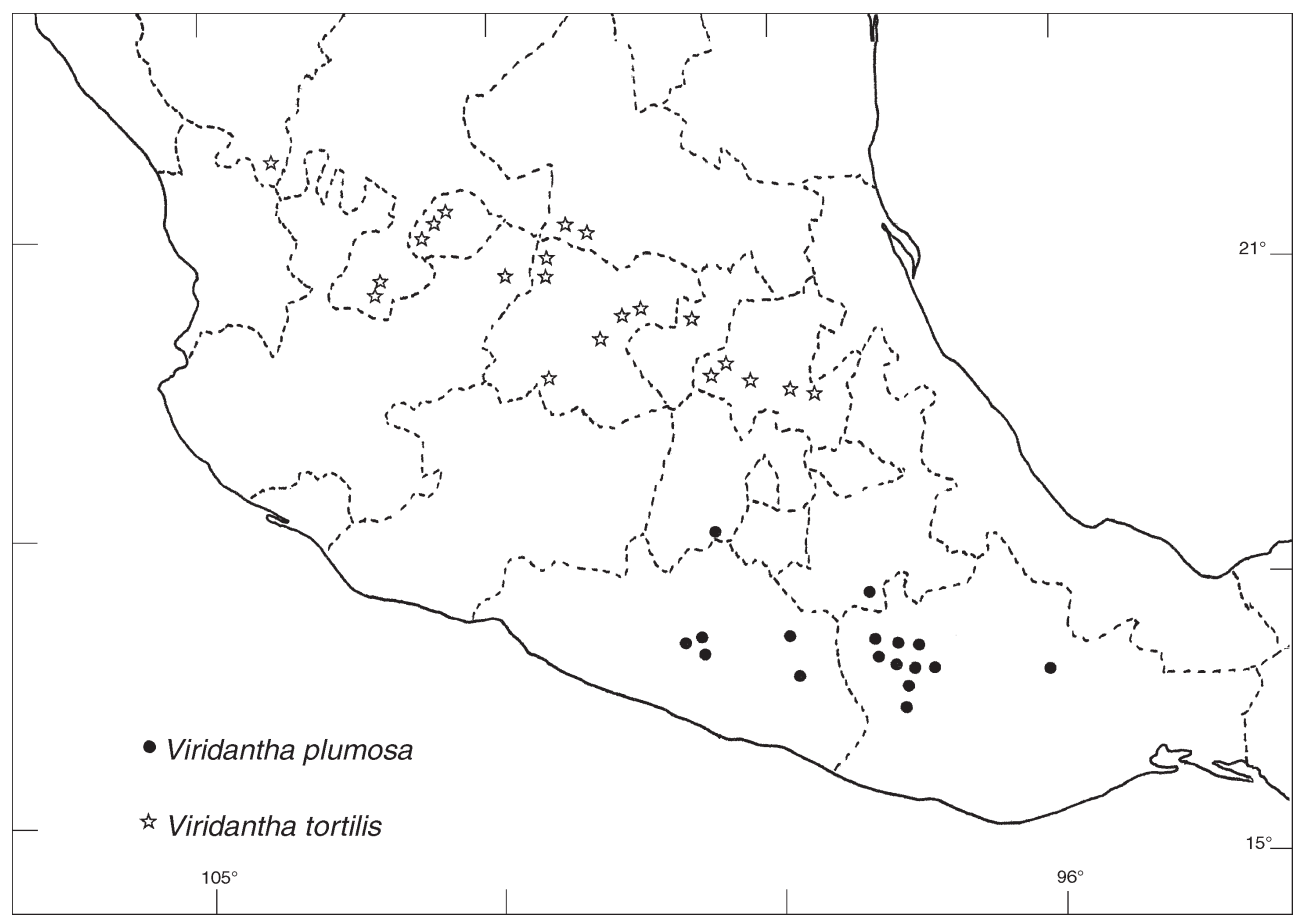

Fig. 5. Distribución conocida de Viridantha plumosa (Baker) Espejo y V. tortilis (Klotzsch ex Baker) Espejo.

[San Miguel] Regla "Rugla", C. Ehrenberg 860 (Holotipo: B?; Isotipos: GH!, MEXU 7636!, MEXU 169260!, VT!).

Tillandsia tortilis Klotzsch ex Beer, Fam. Bromel. 266. 1857, nomen nudum. Platystachys tortile Beer, Fam. Bromel. 266. 1857, pro syn., nomen nudum. TIPO: ?

Tillandsia ehrenbergiana Klotzsch ex Baker, Handb. Bromel. 169. 1889, pro parte, nomen superfluum (C. C. Parry \& Palmer 872).

Tillandsia ehrenbergii Mez, in A. DC., Monogr. Phan. 9: 727. 1896, pro parte, nomen superfluum (C. C. Parry \& Palmer 872).

Tillandsia tortilis Klotzsch ex Baker ssp. curvifolia Ehlers \& Rauh, J. Bromeliad Soc. 40: 167-168, f. 7, 8. 1990. TIPO: Guanajuato, apud Guanajuato, 2200-2400 m, 6.III.1985, R. \& K. Ehlers M 850201 (Holotipo: WU).

V. tortilis se conoce de los estados de Aguascalientes, Durango, Guanajuato, Hidalgo, Jalisco, Querétaro, San Luis Potosí y Zacatecas. Fig. 5.

Esta especie, con una complicada historia taxonómico nomenclatural, fue durante largo tiempo conocida como Tillandsia ehrenbergii, nombre con el cual se encontraba 
identificada en la mayoría del material de herbario revisado. Sin embargo, Till (1986) aclaró la identidad del taxon, demostrando que el nombre correcto para el mismo debe ser T. tortilis.

\section{AGRADECIMIENTOS}

Quiero expresar mi gratitud a Ana Rosa López-Ferrari por su apoyo en el desarrollo del trabajo. Jerzy Rzedowski, Graciela Calderón de Rzedowski, Jacqueline Ceja, Walter Till y Sue Sill leyeron el manuscrito y lo enriquecieron con sus comentarios. Fernando Chiang revisó el artículo, en especial las cuestiones nomenclaturales y el latín. Las ilustraciones fueron realizadas por Rolando Jiménez Machorro. A los curadores de los herbarios revisados, agradezco las facilidades que me otorgaron para consultar las colecciones o, en su caso, el préstamo del material tipo.

Los resultados de este trabajo forman parte del proyecto de tesis de doctorado del autor.

\section{LITERATURA CITADA}

Baker, J. G. 1889. Handbook of the Bromeliaceae. George Bell \& Sons. Londres. 243 pp.

Brown, G. K. \& A. J. Gilmartin. 1984. Stigma structure and variation in Bromeliaceae -neglected taxonomic characters. Brittonia 36(4): 364-374.

Gardner, C. S. 1982. A systematic study of Tillandsia subgenus Tillandsia. Ph. D. Dissertation. Texas A\&M University, College Station. Corpus Christi. 305 pp.

Gardner, C. S. 1986. Preliminary classification of Tillandsia based on floral characters. Selbyana 9: 130-146.

Grant, J. R. 1995. Bromelienstudien. The resurrection of Alcantarea and Werauhia, a new genus. Akad. Wiss. Abh. Math.-Naturwiss. Kl. Trop. Subtrop. Pflanzenwelt. 91: 1-57.

Mez, C. 1896. Bromeliaceae. In: De Candolle, A. Monographiae Phanerogamarum 9: 1-990.

Smith, L. B. \& C. S. Pittendrigh. 1953. Realignments in the Bromeliaceae subfamily Tillandsioideae. J. Wash. Acad. Sci. 43: 401-404.

Smith, L. B. \& R. J. Downs. 1977. Tillandsioideae (Bromeliaceae). Flora Neotropica Monograph 14(2): $1-1492$.

Spencer, M. A. \& L. B. Smith. 1993. Racinaea, a new genus of Bromeliaceae (Tillandsioideae). Phytologia 74(2): 151-160.

Till, W. 1986. Was ist Tillandsia ehrenbergii. ? Die Bromelie 3/86: 39-41.

Till, W. 2000a. Tillandsioideae. In: Benzig, H. Bromeliaceae, profile of an adaptive radiation. Cambridge University Press. Cambridge. pp. 555-572.

Till, W. 2000b. Tillandsia and Racinaea. In: Benzig, H. Bromeliaceae, profile of an adaptive radiation. Cambridge University Press. Cambridge. pp. 573-586. 